\title{
Caso anatomopatológico: causa de muerte súbita en gente joven y revisión de la bibliografía
}

\author{
Bullón-Alcalá Sergio Roberto*, Sosa-Morales Víctor**, Montiel- Esparza Ana Cecilia**
}

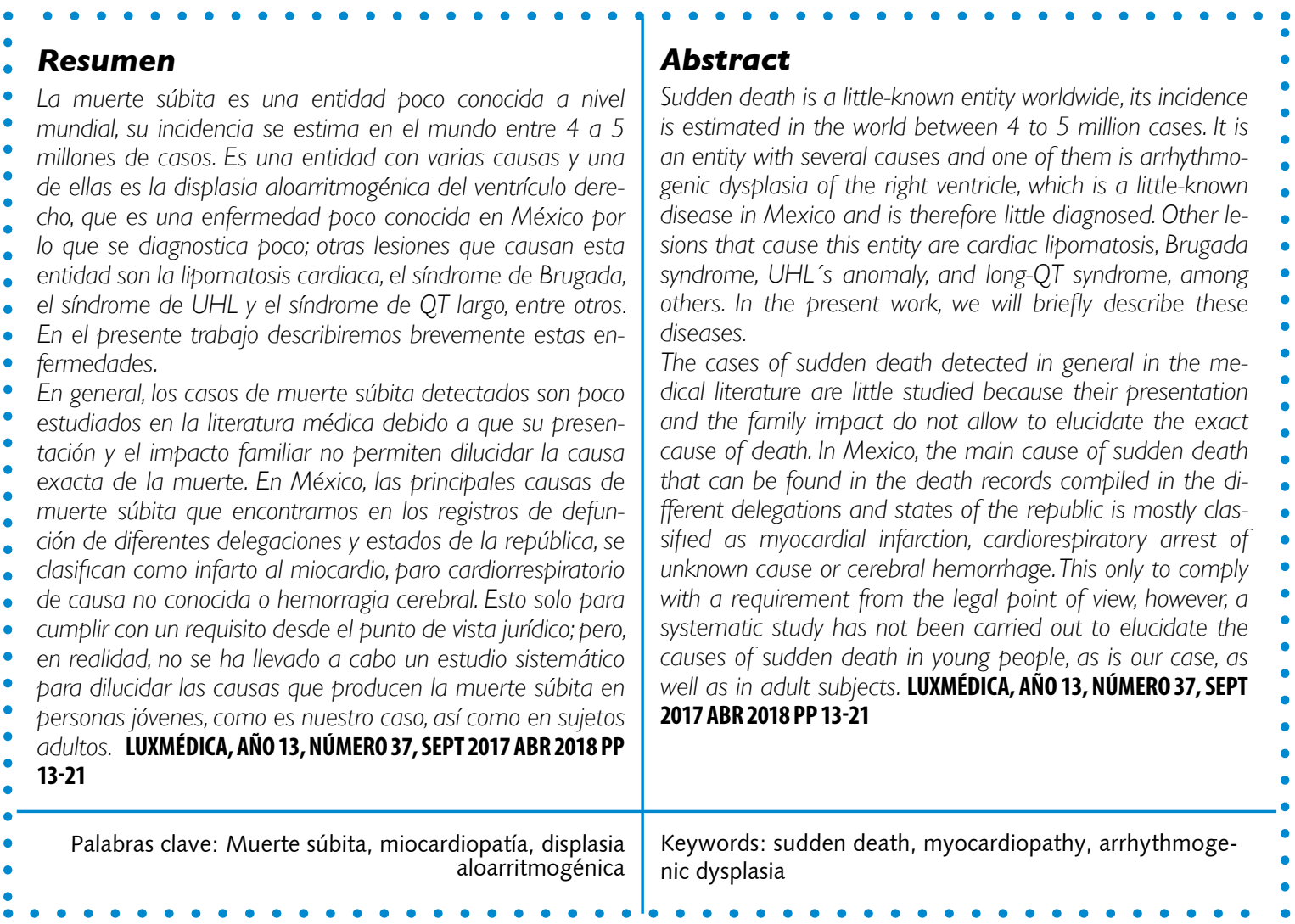

\footnotetext{
Bullón-Alcalá Sergio Roberto. Servicio de anatomía patológica del HGZ 1 IMSS Aguascalientes. Patólogo forense. imsspatologiaags@hotmail.com Teléfono 044-449-180-51-30

** Estudiante del séptimo semestre de la carrera de Medicina del Centro de Ciencias de la Salud de la Universidad Autónoma de Aguascalientes, México. Prosector de autopsia. Correo electrónico vsm_11@hotmail.com

** Estudiante del séptimo semestre de la carrera de Medicina del Centro de Ciencias de la Salud de la Universidad Autónoma de Aguascalientes, México. Prosector de autopsia. Correo electrónico cecymontiel@hotmail.com

Fecha de recibido 15 de diciembre 2017

Fecha de aceptación: 15 de enero 2018

Correspondencia: Dr Sergio Roberto Bullón Alcalá. Servicio de Anatomía Patológica del Hospital General de Zona \# 1 del Instituto Mexicano del Seguro Social. Blvd. José María Chávez 1202, Lindavista, código postal 20270 Aguascalientes, Ags., México. Teléfono 01449913 9022. Correo electrónico: imsspatologiaags@hotmail.com
} 


\section{Introducción}

En la actualidad, el diagnóstico definitivo de la muerte súbita en jóvenes sigue siendo un reto a nivel mundial, pues es una entidad poco conocida, además de que su forma de presentación imita a la de otras condiciones. Sabemos que la autopsia es el estudio sistemático de los diferentes órganos y sistemas del cuerpo humano y que, con el paso de los años, su práctica se ha reducido significativamente, tanto en los hospitales de sector salud, como en los hospitales privados. Ello se debe a diversos factores, incluyendo los elevados costos de dicho estudio. Por tal motivo, es difícil saber con exactitud la causa verdadera de la muerte súbita. En el presente caso se trató de un joven, sin antecedentes de importancia, que presentó muerte súbita.

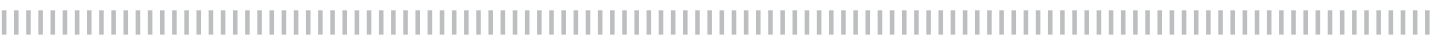

\section{Presentación del caso clínico}

Se trató de un hombre de 19 años de edad que, de acuerdo a lo que refirieron sus familiares, empezó a sentir súbitamente alteraciones en el ritmo respiratorio, motivo por el cual fue trasladado al hospital y en el servicio de urgencias falleció. Por lo anterior y ante el cuadro súbito que desencadenó su fallecimiento, los familiares decidieron que se realizara la necropsia.

\section{Hallazgos de autopsia}

En el servicio de anatomía patológica se recibió el cuerpo de un individuo del sexo masculino de edad aparente a la referida, mesomórfico. A la inspección externa, no se encontraron alteraciones en tegumentos ni mucosas. Al realizar la apertura por cavidades, a nivel del encéfalo se encontró exclusivamente congestión de vasos leptomeníngeos; macroscópicamente, en los cortes coronales no se encontraron lesiones degenerativas ni neoplásicas; asimismo, no se observaron signos de herniación cerebral de ningún tipo. Lo que llamó la atención, fue la presencia de infiltración adiposa en el corazón, con predominio en región epicárdica del ventrículo derecho; en los cortes coronales se apreció adelgazamiento de la pared del ventrículo derecho y presencia de infiltración adiposa transmural; el saco pericárdico no presentó anormalidades. En ambos pulmones, tráquea y bronquios no se encontraron alteraciones. No se encontraron objetos que obstruyeran la vía respiratoria superior ni inferior; al corte, el parénquima pulmonar no presentó ninguna alteración. En la exploración del tubo digestivo, páncreas, hígado y bazo, tracto urinario y glándulas suprarrenales no se encontraron alteraciones. Se realizaron cortes histológicos de todos los órganos.

La única alteración significativa en la necropsia fue la presencia de depósito graso a nivel ventricular derecho. El peso del corazón fue de 325 gramos; se realizó la disección del corazón de acuerdo al manual de procedimientos de patología forense, a nivel de ventrículo derecho se observó presencia de tejido adiposo en el espesor de la pared ventricular como único hallazgo de interés. La presencia aislada de tejido adiposo en el corazón es un hallazgo frecuente encontrado en las necropsias, siendo ésta más aparente en individuos ancianos, así como en personas obesas. Hay que 
recordar que en el corazón normal existe cierta cantidad de grasa a nivel cardiaco en las regiones anterolateral y apical del ventrículo derecho. También debemos recordar que puede haber tejido adiposo normal a nivel de las bandas musculares intramurales.

En los cortes microscópicos no se encontraron alteraciones inflamatorias, degenerativas ni neoplásicas en la víscera cardiaca. Los cortes en el ventrículo derecho mostraron la presencia de depósito de colágena y tejido adiposo entre los cardiocitos, que se evidenció con la tinción tricrómica de Masson (ver corte histológico figura 2).

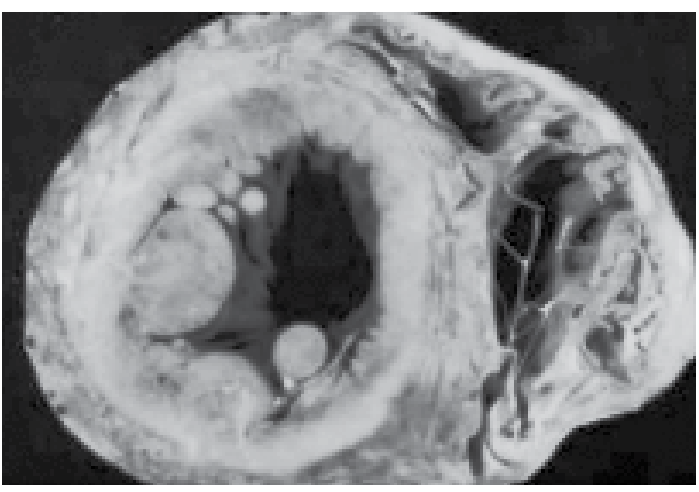

Figura 1. Corte coronal de corazón: presencia de infiltración grasa panmural con adelgazamiento de su pared libre.

Por lo anterior, se concluyó que la causa del fallecimiento fue una miocardiopatía arritmogénica o displasia arritmogénica del ventrículo derecho, que condujo a una muerte súbita consecutiva a una fibrilación ventricular.

\section{Miocardiopatía arritmogénica del ventrículo derecho} Introducción

La miocardiopatía arritmogénica del ventrículo derecho (MAVD) es un desorden del músculo cardíaco caracterizado por un reemplazo fibroadiposo progresivo del miocardio del ventrículo derecho, inicialmente regional y más tarde global, incluso con afección del ventrículo izquierdo en las formas más graves.

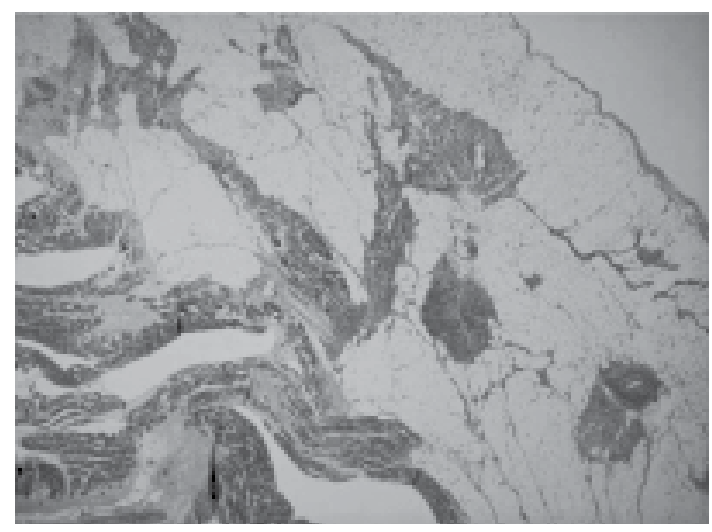

Figura 2. Corte microscópico de la pared del ventrículo derecho mostrando zonas de fibrosis con atrapamiento de los cardiocitos. Tinción tricrómica de Masson (40x).

La prevalencia de la MAVD en la población general es difícil de estimar debido a las dificultades que comporta el diagnóstico. Los estudios realizados en Europa indican una prevalencia de entre 0.6 y $4.4 \%$, pero es posible que estas cifras estén sesgadas por las diferencias geográficas existentes en la disponibilidad de expertos clínicos y anatomopatológicos. ${ }^{1,2}$

\section{Genética}

La displasia arritmogénica del ventrículo derecho se ha descrito y aceptado como una enfermedad genéticamente determinada, debido a que entre un $30 \%$ y $50 \%$ de los fallecidos por esta causa refieren antecedentes familiares de muerte súbita a edades tempranas ${ }^{3}$. El patrón hereditario es del tipo autosómico dominante, con pe- 
netrancia incompleta y expresividad variable. La forma autosómica recesiva también se ha descrito asociada a la enfermedad de Naxos y a mutaciones en el gen codificador de la desmoplaquina (DSP). ${ }^{4}$ La forma autosómica recesiva se caracteriza por la miocardiopatía arritmogénica del ventrículo derecho, queratodermatitis palmoplantar y el cabello rizado típico.

Las investigaciones genéticas han centrado su objetivo en siete genes identificados asociados a la displasia arritmogénica del ventrículo derecho, entre los que se encuentran cuatro que codifican proteínas del desmosoma (proteínas de unión intercelular), estos son: plakoglobina (JUP), desmoplakina (DSP), plakofilina-2 (PKP2), desmogleina-2 (DSG2), desmocollina-2 (DSC2), factor de crecimiento transformador beta-3 (transforming growth factor beta-3, TGF $\beta-3$ ) y TMEM 43 . El $45 \%$ de los pacientes presentan una mutación que afecta a la proteína plakofilina 2 (PKP2). ${ }^{3}$

\section{Patogénesis}

Es una enfermedad del músculo cardíaco caracterizada por pérdida de miocardiocitos y sustitución de los mismos por tejido conectivo denso o fibroadiposo, que puede dar lugar a arritmias, muerte súbita e insuficiencia cardíaca. ${ }^{5}$ La sustitución progresiva del miocardio por células del tejido adiposo y del tejido conectivo denso sucede tras un exagerado e inadecuado proceso de apoptosis, y aunque la causa de la MAVD es aún desconocida, se plantean varias teorías. ${ }^{3}$

En la teoría inflamatoria, apoyada por la aparición de infiltrados inflamatorios en series necrópsicas, la afección miocárdica vendría explicada por un proceso continuado de daño y reparación simulando una miocarditis crónica. En la teoría genética, las mutaciones en genes que codifican proteínas específicas darían lugar a la "distrofia miocárdica"; en este sentido, los recientes descubrimientos de mutacio- nes causales de la enfermedad inducen a esbozar nuevas teorías patogénicas basadas en el estrés mecánico intercelular. Los estudios descriptivos señalan que la sustitución progresiva del miocardio por células del tejido adiposo y del tejido conectivo denso sucede tras un exagerado e inadecuado proceso de apoptosis. Los modelos animales apoyan el desequilibrio provocado por el estrés mecánico intercelular como un desencadenante de la apoptosis. ${ }^{4}$

Las proteínas más estudiadas en la MAVD son la plakoglobina y la desmoplaquina. La primera es una proteína citoplasmática que participa en la unión de los filamentos intermedios y el citoesqueleto de la actina con los complejos transmembrana que conectan con los complejos adyacentes. Por otra parte, la desmoplaquina ancla los filamentos intermedios a la membrana plasmática y constituye una base para el mantenimiento de la integridad celular; por lo que, un error en la codificación de alguna de ellas llevará a un sustrato inestable. ${ }^{6,7}$

Se han puntualizado seis mutaciones en el receptor cardiaco de la rianodina 2, las que generarían un aumento en la concentración citosólica de calcio, lo que las hace proapoptósicas. ${ }^{6}$ El receptor cardíaco de la rianodina (RYR2) forma parte de la estructura que regula los canales del calcio del retículo sarcoplásmico. El funcionamiento correcto de estos canales es fundamental para el acoplamiento excitación-contracción y la homeostasis del calcio en los miocitos. ${ }^{4}$ En estas familias se describe una susceptibilidad mayor a las taquicardias ventriculares inducidas por el ejercicio.

En 1995, Rampazzo describió un segundo locus asociado a lo que él denomina una variante nueva de MAVD, la MAVD2.4,8 Ésta se caracteriza por presentar taquicardias ventriculares inducidas tras estímulo catecolaminérgico. En particular, las taquicardias inducidas por el ejercicio se perfi- 
lan como una entidad independiente de la MAVD. ${ }^{4}$ De este modo, aumentaría la propensión a arritmias ventriculares en situaciones de exposición a las catecolaminas, especialmente durante el ejercicio. ${ }^{4,9}$

\section{Presentación clínica}

La presentación clínica de la miocardiopatía arritmogénica del ventrículo derecho, como se ha descrito hasta ahora, es variable. En primer lugar, en un gran número de pacientes la enfermedad es silente y la primera manifestación es la muerte súbita. ${ }^{3}$

Las manifestaciones de MAVD se pueden presentar a cualquier edad, pero generalmente son jóvenes o de mediana edad (33 \pm 14 años); la mayoría de los pacientes son hombres (proporción 3:1). Debido a la progresiva naturaleza del trastorno, los pacientes pueden debutar con varios síntomas, siendo más comunes la fatiga y el síncope, y menos frecuentes la insuficiencia cardíaca derecha o la biventricular que simulan una miocardiopatía dilatada..$^{5,9}$ Las arritmias ventriculares, que son tan frecuentes y le dan el nombre a la enfermedad, varían desde la simple extrasistolia ventricular hasta la taquicardia o la fibrilación ventricular. Las taquiarritmias supraventriculares, las extrasístoles o la fibrilación auricular son también frecuentes. La muerte súbita predomina durante el esfuerzo, aunque se han descrito casos de muerte durante el sueño. ${ }^{10}$

Se pueden describir cuatro estadíos de la enfermedad:

1) Fase temprana o silente: por lo general, es asintomática y su manifestación más común es la muerte súbita.

2) Fase inestable con predominio de arritmias sintomáticas: por lo general, se evidencian con bloqueo completo de rama izquierda.

3) Fase de fallo ventricular derecho con relativa conservación del ventrículo izquierdo.
4) Fase final: hay dilatación biventricular, que en ocasiones es indistinguible de la miocardiopatía dilatada, tiende a complicarse con tromboembolismos y fibrilación atrial. ${ }^{3}$

\section{Anatomía patológica}

Macroscópicamente, se aprecian importantes anomalías estructurales del ventrículo derecho: reemplazo graso transmural del miocardio de la pared libre del ventrículo, más evidente en la región subepicárdica, y miocardio adelgazado y frecuentemente dilatado formando aneurismas saculares, que suelen ubicarse en el ápex, en el infundíbulo o en la pared postero-lateral, lo que se conoce como "el triángulo de la displasia". Ver figura 2.

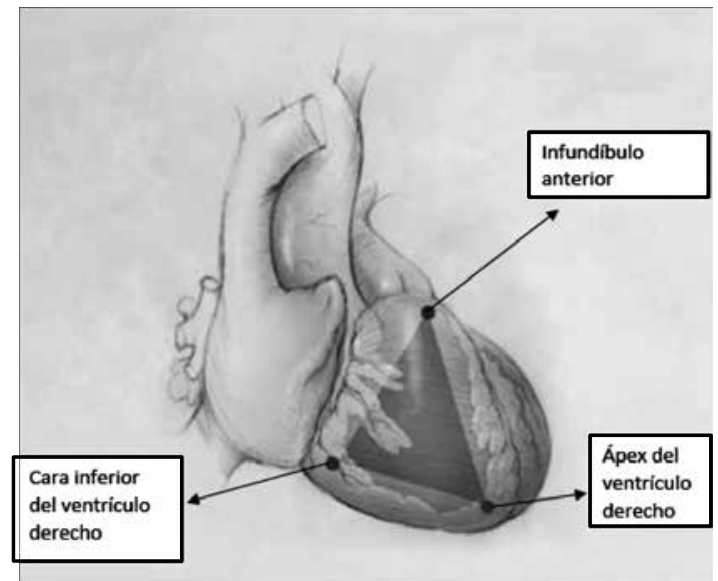

Figura 3. Triángulo de la displasia.

No hay, sin embargo, un incremento significativo de la grasa epicárdica. En el área afectada, se puede formar un agujero electrofisiológico que puede potencialmente convertirse en un sustrato para las arritmias de reentrada ${ }^{9}$. Además, se observa un ventrículo derecho dilatado y se puede apreciar un adelgazamiento de la pared libre sin llegar a haber contacto directo entre epicardio con endocardio ${ }^{3}$.

La microscopía pone de manifiesto la característica infiltración grasa y el reemplazo fibroadiposo del miocardio. Se aso- 
cian dos datos histológicos constantes y diferenciales: la atrofia de miocitos, que sufren degeneración vacuolar con picnosis nuclear y pérdida de miofibrilla; y el infiltrado inflamatorio linfocitario de tipo CD4 (el hallazgo en la biopsia de linfocitos, se considera un factor agravante ya que se asocia a mayor riesgo arrítmico), con focos de necrosis, que se considera la forma inicial de la enfermedad en que se producen miocarditis recidivantes o crónicas que evolucionarán a la cicatrización con fibrosis. ${ }^{11,12}$ Por otra parte, para evitar los falsos positivos, la muestra de la biopsia debe cumplir tres criterios fundamentales que son la presencia de más de un

\begin{tabular}{|l|}
\hline SIGNOS Y SINTOMAS DE MAVD \\
\hline Síntomas \\
\hline Dolor abdominal \\
\hline Disminución de la tolerancia al ejercicio \\
\hline Mareo \\
\hline Disnea (especialmente al hacer esfuerzo) \\
\hline Fatiga \\
\hline Confusión mental \\
\hline Palpitaciones \\
\hline Síncope o desmayo \\
\hline Signos \\
\hline Paro cardiaco \\
\hline Edema periférico \\
\hline Muerte súbita \\
\hline Taquicardia \\
\hline
\end{tabular}

$3 \%$ de grasa, más de un $40 \%$ de tejido conectivo denso y menos de un $45 \%$ de miocitos. Por tanto, una infiltración grasa no es sinónimo de $M A V D,{ }^{12}$ la cantidad de miocitos residuales en el tejido debe ser menor a $45 \%$, si es de 45 a $70 \%$ es una forma limítrofe de MAVD, pero si es mayor del $70 \%$ este diagnóstico puede ser excluido. ${ }^{6}$ La afección cardiaca llega a ser difusa (incluyendo ventrículo izquierdo) hasta en el $75 \%$ de los casos, y son los hallazgos típicos: el adelgazamiento de la pared miocárdica, la aparición de cicatrices y las dilataciones aneurismáticas. ${ }^{12}$

\section{Diagnósticos diferenciales}

Existen diversas condiciones que causan muerte súbita, entre las que podemos encontrar el síndrome de $\mathrm{UHL}$, que es una cardiopatía congénita rara, caracterizada por la ausencia casi total de miocardio en la pared libre del ventrículo derecho; las válvulas tricúspide y pulmonar se mantienen morfológicamente normales; ${ }^{13}$ otra entidad es la denominada metaplasia grasa post infarto que es un hallazgo histológico frecuente en los infartos de miocardio antiguos, el miocardio infartado es sustituido por fibrosis cicatricial y posteriormente ocupado por adipocitos.

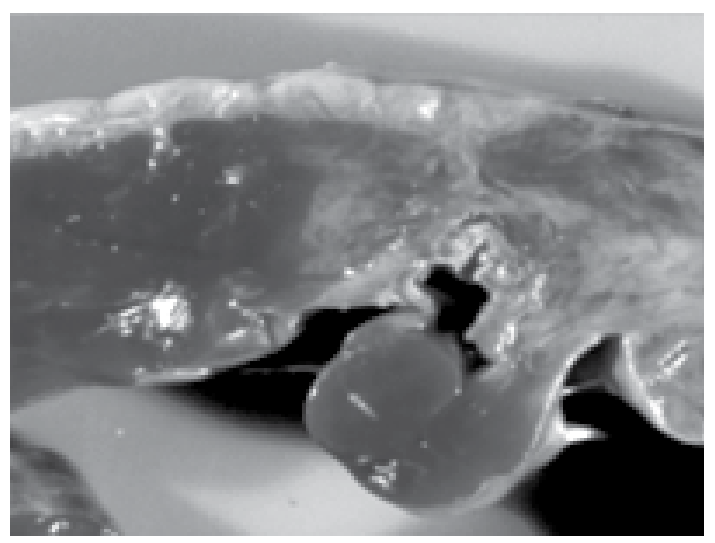

Figura 4. Fotografía macroscópica casos de autopsia. Infiltración grasa post-infarto.

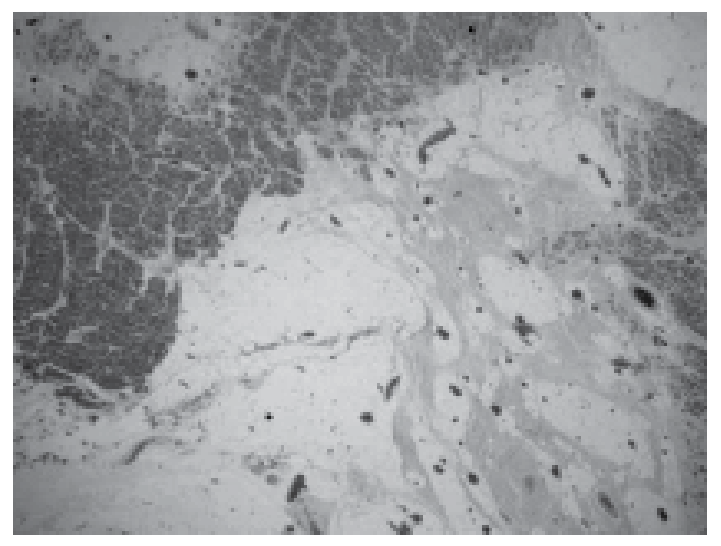

Figura 5. Fotomicrografía de la pared libre del ventrículo izquierdo cara anterior, en la cual se observa necrosis de los cardiocitos, presencia de material colágeno de tono verde tenue y tejido adiposo. (40X HE) 


\section{Lipomatosis cardíaca}

La lipomatosis cardiaca es un proceso degenerativo en el que las células miocárdicas son sustituidas por grasa, afectando en mayor medida al ventrículo derecho (VD). Hasta un $50 \%$ de las personas ancianas presenta cierto grado de lipomatosis cardiaca. ${ }^{14}$ Se manifiesta en sujetos sin antecedentes patológicos familiares, ya que es clínicamente estable. Se trata de un aumento difuso de grasa intramiocárdica transmural de las regiones antero-lateral e infundibular del VD y de la grasa epicárdica, con un ventrículo izquierdo (VI) habitualmente normal. La pared del VD afectado presenta un grosor normal o ligeramente hipertrófico, es decir, no hay pérdida de miocitos.

Los pacientes permanecen durante años asintomáticos, pero puede desencadenar muerte súbita, atribuida a la infiltración del miocardio y del nodo auriculoventricular. ${ }^{14}$

En el estudio microscópico es importante resaltar que no existen datos de inflamación, los cardiomiocitos tienen aspecto normal y no hay áreas de fibrosis cicatricial. Se trata, por tanto, de un área poco arritmogénica, a diferencia de la displasia arritmogénica del VD, en la que hay un reemplazo de los cardiomiocitos por tejido adiposo y/o fibroadiposo, acompañado de cambios degenerativos de los miocardiocitos localizados entre el tejido adiposo, y de la metaplasia tras infarto de miocardio antiguo, en la que hay sustitución del colágeno cicatricial por adipocitos. ${ }^{14}$

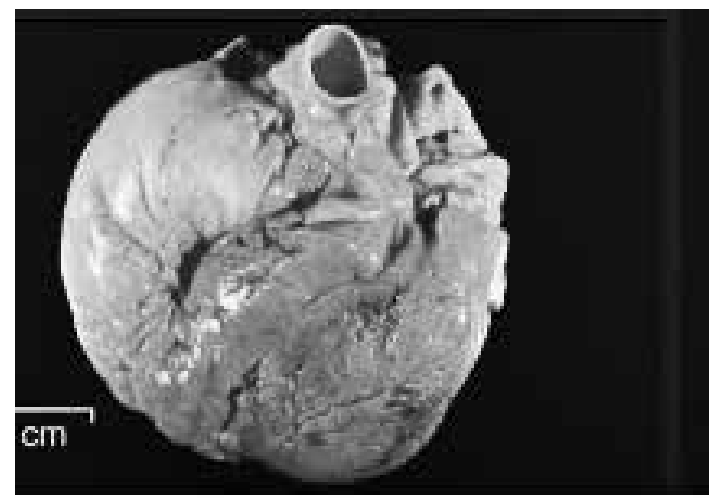

Figura 6. Lipomatosis cardiaca, caso de autopsia.

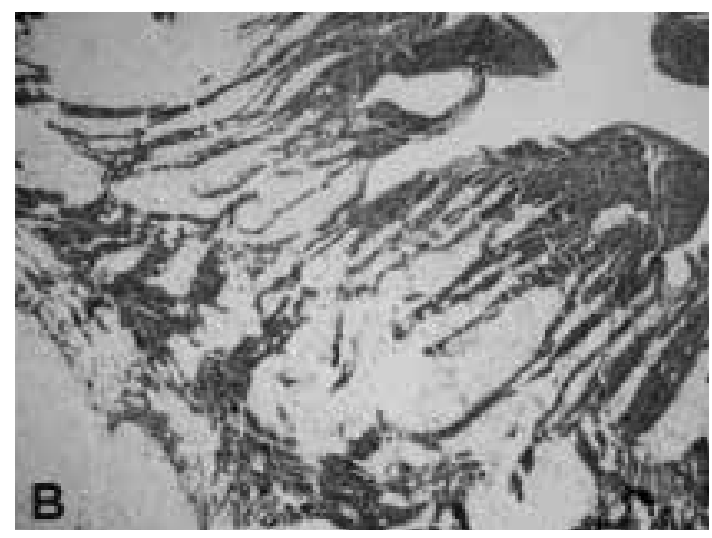

Figura 7. Imagen microscópica de la pared libre del ventrículo izquierdo mostrando la presencia de fibras miocárdicas las cuales se encuentran rechazadas por la infiltración de tejido adiposo. No hay evidencia de proceso inflamatorio ni fibrosis. Caso de autopsia. (40X HE)

A continuación, se muestra una correlación entre lipomatosis cardiaca, MAVD y metaplasia lipomatosa.

\section{Tabla I}

\begin{tabular}{|c|c|c|}
\hline $\begin{array}{l}\text { LIPOMATOSIS } \\
\text { CARDIACA }\end{array}$ & MAVD/D & $\begin{array}{l}\text { METAPLASIA } \\
\text { LIPOMATOSA }\end{array}$ \\
\hline Edades avanzadas & Jóvenes & Edades avanzadas \\
\hline $\begin{array}{l}\text { No hay predominio } \\
\text { de sexo }\end{array}$ & Varones & Varones \\
\hline $\begin{array}{c}\text { No antecedentes } \\
\text { personales }\end{array}$ & $\begin{array}{l}\text { Historia clínica de } \\
\text { arritmias y/o síncope }\end{array}$ & $\begin{array}{c}\text { Antecedentes de } \\
\text { enfermedad coronaria } \\
\text { y tratamientos espe- } \\
\text { cíficos }\end{array}$ \\
\hline $\begin{array}{l}\text { No antecedentes } \\
\text { familiares }\end{array}$ & $\begin{array}{l}\text { Antecedentes familiares } \\
\text { de muerte súbita }\end{array}$ & \\
\hline $\begin{array}{l}\text { Aumento de grasa } \\
\text { epicárdica }\end{array}$ & $\begin{array}{l}\text { No aumento de grasa } \\
\text { epicárdica }\end{array}$ & $\begin{array}{l}\text { No aumento de grasa } \\
\text { epicárdica }\end{array}$ \\
\hline $\begin{array}{l}\text { Afectación predomi- } \\
\text { nante delVD }\end{array}$ & $\begin{array}{l}\text { Afectación predominan- } \\
\text { te del VD }\end{array}$ & $\begin{array}{l}\text { Afectación predomi- } \\
\text { nante del VI }\end{array}$ \\
\hline $\begin{array}{l}\text { Miocardio normal } 0 \\
\text { engrosado }\end{array}$ & Miocardio adelgazado & $\begin{array}{c}\text { Miocardio normal } 0 \\
\text { adelgazado }\end{array}$ \\
\hline No inflamación & Reacción inflamatoria & \\
\hline No atrofia celular & Atrofia de miocitos & \\
\hline No fibrosis & Fibrosis & Fibrosis \\
\hline
\end{tabular}

Otro padecimiento similar es la taquicardia ventricular polimórfica catecolaminérgica, la cual es una canalopatía ca- 
racterizada por la inducción de arritmias ventriculares polimórficas en presencia de catecolaminas. Deberá sospecharse en todo paciente joven, en especial niño o adolescente, que presente síncopes relacionados con el ejercicio físico o el estrés emocional, que no tenga cardiopatía estructural y que su electrocardiograma muestre un intervalo QT normal.

\section{Otras condiciones causantes de muerte súbita}

\section{Síndrome de Brugada}

El síndrome de Brugada, descrito por primera vez en 1992, se caracteriza por un patrón electrocardiográfico característico en precordiales derechas y la predisposición a presentar arritmias ventriculares y muerte súbita. ${ }^{15}$

El síndrome del bloqueo de rama derecha, elevación persistente del segmento ST y muerte súbita, conocido como síndrome de Brugada, fue descrito como un nuevo síndrome clínico-electrocardiográfico causante de arritmias ventriculares y muerte súbita en pacientes sin cardiopatía estructural evidente. ${ }^{15}$

El síndrome de Brugada se encuentra catalogado dentro del grupo de las canalopatías, es decir, se trata de un trastorno eléctrico primario secundario a una alteración de la función de los canales iónicos del miocardiocito, en ausencia de cardiopatía estructural y asociado a un significativo aumento del riesgo de muerte súbita en individuos jóvenes, en la tercera y cuarta década de la vida principalmente. ${ }^{16}$

El síndrome de Brugada se transmite característicamente según un patrón de herencia autosómico dominante. No obs- tante, en una proporción significativa de pacientes, la enfermedad puede ser esporádica. ${ }^{15}$

Los pacientes con síndrome de Brugada permanecen en su mayoría asintomáticos. No obstante, se ha descrito que de un 17 a un $42 \%$ de ellos presenta síncope o muerte súbita como consecuencia de una arritmia ventricular en algún momento de su vida. ${ }^{15}$

\section{Síndrome de QT largo}

El síndrome del QT largo (SQTL) es la primera canalopatía arritmogénica descrita y es la mejor estudiada hasta el momento. Puede ser debido a una alteración genética (SQTL congénito) o secundaria a anomalías metabólicas, fármacos, etc., (SQTL adquirido). Su importancia clínica radica en la asociación con una arritmia característica y potencialmente letal: "torsade de pointes" o taquicardia ventricular polimorfa o helicoidal. ${ }^{17}$

La prevalencia del SQTL congénito se estima en 1:5000 y aparece característicamente en jóvenes. La prevalencia exacta del SQTL adquirido es desconocida, puede aparecer a cualquier edad, siendo más frecuente en ancianos polimedicados. Mientras que en el SQTL congénito, el mal funcionamiento de los canales iónicos se debe a mutaciones en los genes que codifican las proteínas que los forman, en el SQTL adquirido es debido a la interferencia, generalmente de fármacos, con las corrientes iónicas de entrada y salida, implicadas en el potencial de acción ventricular. ${ }^{17}$

Las personas que sufren SQTL generalmente permanecen asintomáticas. La sintomatología, en caso de presentarse es muy variable, desde mareos a muerte súbita. ${ }^{17}$

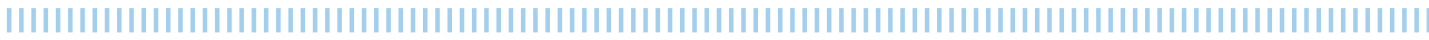

\section{Discusión}

Se define como muerte súbita al fallecimiento de una persona aparentemente sana en un lapso de evolución de una a dos horas.
En México no se tiene registros verdaderos de causas de muerte súbita en gente joven, ya que no se realizan las necropsias debido a diversos factores autóctonos y/o 
similares a los informados en otras partes del mundo. Por tal motivo, cuando se produce una muerte súbita y ante los momentos trágicos por los que pasan los familiares, los médicos que expiden el certificado de defunción en el que por lo regular asientan como causa de muerte un infarto al miocardio y/o paro cardiorrespiratorio; por lo que, sin realizar la necropsia de acuerdo a los estándares estableci- dos, quedan veladas las causas verdaderas del fallecimiento.

Aunado a lo anterior, ante los diagnósticos diferenciales ya mencionados, los estudios de muerte súbita de origen cardiaco han ido avanzando, llegando en la actualidad a estudios de biología molecular, los cuales tienen un costo elevado; que en caso de realizarse la autopsia, dificulta llegar al diagnóstico.

\section{Conclusiones}

La muerte súbita se puede presentar durante todas las épocas de la vida; sin embargo, en la actualidad, ante los avances científicos con los que se cuenta, es importante sensibilizar al sector salud y los familiares para que se realicen todos los procedimientos que permitan identificar con exactitud las diferentes causas de muerte súbita.

Figuras 1, 2, 4, 5 ,6 ,7. Material intelectual, casos de autopsia.

\section{Bibliografía}

1. Giovanni Q. Perry M. Criterios diagnósticos para la miocardiopatía arritmogénica del ventrículo. Rev Esp Cardiol. 2012; 65(7):599-605

2. Eric L. Arrhythmogenic Right Ventricular Dysplasia. American Family Physician. 2006; 73: 1391-1398.

3. Allan L. Miocardiopatía Arritmogénica del Ventrículo. Med. leg. Costa Rica. 2015; 32: 160-166.

4. Estebana M. Pinillab J. McKennaa J. Actualización en miocardiopatía arritmogénica del ventrículo derecho: genética, diagnóstico, manifestaciones clínicas y estratificación de riesgo. Rev Esp Cardiol. 2004;57:757-67

5. Izurieta C. Grasiosi J. Rocchinotti M. Miocardiopatía arritmogénica del ventrículo derecho. Breve revisión de la literatura a propósito de un caso. Arch Cardiol Mex. 2013; 83:282-288

6. Álvarez A. Navas S. Martínez E. et al. Miocardiopatía arritmogénica del ventrículo derecho. MÉD UIS. 2014; $27: 123-134$.

7. Awad M. Calkins H. Judge D. Mechanisms of disease: molecular genetics of arrhythmogenic right ventricular dysplasia/ cardiomyopathy. Nat. Clin. Pract. Cardiovasc. Med. 2008; 5:258- 67.

8. Rampazzo A. Nava A. Erne P. Eberhard M. Vian E. Slomp $P$. et al. A new locus for arrhythmogenic right ventricular cardiomyopathy (ARVD2) maps to chromosome 1q42q43. Hum Mol Genet. 1995; 4: 2151-4.

9. Wichter T. Schafers M. Rhodes CG. Borggrefe M. Lerch H. Lammertsma AA. et al. Abnormalities of cardiac sympathetic innervation in arrhythmogenic right ventricular cardiomyopathy: quantitative assessment of presynaptic norepinephrine reuptake and postsynaptic beta-adrenergic receptor density with positron emission tomography. NCBI Circulation. 2000; 101:1552-8.

10. Albina G. Laíño R. Ginger A. Displasia arritmogénica del ventrículo derecho: revisión de una enfermedad, poco común con un espectro variado de presentaciones clínicas. Electrofisiología Y Arritmias. 2009; 2:1-72.

11. Corrado D. Link M. Calkins H. Arrhythmogenic Right Ventricular Cardiomyopathy. New England Journal of Medicine. 2017; 376:1-72.

12. Francisco J. Bodegas A. Gomez C. Arana J. Barrenetxea J. , Miocardiopatía arritmogenica de ventrículo derecho. Gac Med Bilbao. 2007; 104: 70-74.

13. Yabur-Espitia M. Muñoz-Castellanos L. Espinola-Zavaleta N. Enríquez-Rodríguez E. Alcantar-Fernández A. Anomalía de Uhl: Informe de un caso con correlación anátomo-ecocardiográfica. Arch Cardiol Mex 2010;80(2):108-112.

14. Zamarrón-de Lucas E. García-Fernández E. Carpio C. Alcolea S. Martínez-Abad Y. Álvarez-Sala R. Lipomatosis cardiaca masiva, hallazgo en la autopsia de un paciente con muerte súbita. Medicina Clinica (Barc). 2016;146(12):541-543.

15.- Benito B. Brugada J. Brugada R. Brugada P. Síndrome de Brugada. Rev Esp Cardiol. 2009;62(11):1297-315.

16.- Picón R. Síndrome de Brugada: puesta al día, Cardiocore, 2013;48(2):69-74.

17.- Blanco A. López I. Cobas M. Síndrome de QT largo. Cad Aten Primaria 2010; 17:40-43. 